\title{
Improvement in light-extraction efficiency of light emitting diode using microlenses fabricated by a novel and cost-effective method
}

\author{
RAHUL K SHARMA ${ }^{1,2, *}$, MONICA KATIYAR ${ }^{1,2}$ and DEEPAK ${ }^{1,2}$ \\ ${ }^{1}$ Department of Materials Science and Engineering, Indian Institute of Technology Kanpur, Kanpur 208016, India \\ ${ }^{2}$ Samtel Centre for Display Technologies, Indian Institute of Technology Kanpur, Kanpur 208016, India \\ *Author for correspondence (rahul.physics.iitd@gmail.com)
}

MS received 7 November 2016; accepted 27 June 2017; published online 2 February 2018

\begin{abstract}
A low cost, solution-based novel process was proposed, which avoids any photo-lithographically fabricated $\mathrm{Si}$ template and yet yields small dimension microlenses. The microlenses were fabricated using a well-known chemical technique called dewetting of polymer thin film. Polystyrene (PS) was used to make a polymer thin film with thickness ranging from 20 to $40 \mathrm{~nm}$. Then, this PS film was kept in dewetting solution namely methylethylketone (MEK) to obtain the microlenses. Dimension of these microlenses was measured to be the order of 1-2 $\mu \mathrm{m}$. The effect of various microlens parameters such as diameter and area fraction on light-extraction efficiency was systematically studied. Improvement of $4 \%$ in extraction efficiency was obtained by employing it on white light emitting diode. The area fraction of microlenses was increased up to 0.34 by reducing the spin speed. The light-extraction efficiency was further enhanced up to $7 \%$ upon increasing the area fraction of microlenses.
\end{abstract}

Keywords. Organic polymer; thin films; dewetting; microlenses; luminescence; light-extraction efficiency.

\section{Introduction}

The external quantum efficiency (EQE) of an organic light emitting diode (OLED) can be enhanced by using lightextraction techniques. Till now, various light-extraction techniques were reported in the literature to enhance the efficiency of an OLED. These light-extraction techniques can be classified into two categories, namely, external and internal device modification techniques [1]. The use of microlens array to enhance the light-extraction efficiency of an OLED is one of the most efficient techniques without adversely affecting electrical characteristics of the device.

The effect of microlens array on outcoupling efficiency of OLEDs was first observed by Moller and Forrest [2]. They fabricated microlens array with $10 \mu \mathrm{m}$ diameter, arranged in a square geometry, using an elastomeric compound polydimethylsiloxane (PDMS). The improvement in the efficiency by a factor of 1.5 was measured. Since the beginning, various geometries of microlens array were tried to study their effect on the light extraction efficiency. Further, microlenses with different shapes namely hemispherical [2-10], square-shaped [11-13], cylindrical [14] and elliptical [15] were tested.

In general, the microlens array is fabricated using conventional method of photolithography. Even though photolithography is a well-established and extensively used technique for microlens fabrication, it has some pitfalls such as: (i) control of etching parameters is difficult for the formation of submicron-size microlenses; (ii) it is a complex process as it involves large number of steps, starting from substrate cleaning to microlens fabrication; (iii) etching process for $\mathrm{Si}$ and $\mathrm{SiO}_{2}$ involves use of hazardous chemicals; (iv) annealing of elastomeric material PDMS can have adverse effects on the transmittance of the microlenses, which can degrade the device performance; and (v) above all, it is an expensive and time-consuming process.

From the points mentioned above, it can be inferred that fabrication of microlenses by lithography using Si template is not a cost-effective process. Therefore, there is a need to develop a simple and cost-effective process for fabrication of microlenses. To establish such a process, a solution-based, economically viable and novel process is proposed which avoids photo-lithographically fabricated Si templates and yet yields microlenses with smaller dimensions. This process is called dewetting of polymer thin films. In this paper, we are elaborating one of the most exciting uses of the dewetting process, which was not reported till date.

Thickness of polymer film plays an important role in wetting properties of the substrate along with surface energy and interfacial energy. When thickness of polymer films is $>100 \mathrm{~nm}$, interfacial and gravitational forces prevail over van der Waals forces, making these films stable. But, in case of polymer films with thickness $<100 \mathrm{~nm}$, inter-surface interaction and van der Waals forces dominate, which destabilize the film. This destabilization of thin film results in spontaneous fragmentation of thin film into an array of holes or droplets due to instability [16,17]. These droplets or holes are called microlenses. The Navier Stokes equation for a polymer thin film with the influence of intermolecular 
forces on substrate exhibiting long-wave instability can be mathematically represented as follows:

$$
\begin{gathered}
3 \mu h_{t}+\left[(h-a f)^{3}\left\{\gamma\left(h_{x x}+h_{y y}\right)-\phi\right\}_{x}\right]_{x} \\
+\left[(h-a f)^{3}\left\{\gamma\left(h_{x x}+h_{y y}\right)-\phi\right\}_{y}\right]_{y}=0,
\end{gathered}
$$

where $h=h(x, y, t)$ is a local film thickness, $a f(x, y)$ is the physical roughness of the substrate $(a=0$ for smooth and homogeneous substrate), $\phi(=\partial \Delta G / \partial h)$ is the effective interface potential per unit volume or conjoining pressure ( $-\phi=$ disjoining pressure), $\Delta G$ is the excess interaction energy per unit area as a function of local thickness. This equation generally examines the stability of the uniform thin film with the mean thickness $h_{\mathrm{o}}$. If any surface has a small periodic perturbation such that it can be represented in terms of wave vector $k$ by the given equation

$$
h(x, t)=h_{\mathrm{o}}+\varepsilon \sin (k x) \exp (\omega t),
$$

where $\omega$ is initial growth coefficient, which is defined as

$$
\omega=C\left[-\gamma k^{4}-(\partial \phi / \partial h) k^{2}\right],
$$

From the above equations, it is inferred that for perturbation to grow, $\omega$ should posses a positive value, which is possible only when $\partial \phi / \partial h<0$, as the first term in the equation represents the surface tension which is always negative. Thus, when $\partial \phi / \partial h<0$, perturbations in the films will be more and hence, the instability is spontaneous. Even though, this is a promising technique for dewetting, it has two major limitations on pattern resolution and aspect ratio. First limitation is on feature size due to (i) weak van der Waals destabilizing forces and (ii) high surface tension. These factors limit the feature size, which is related to wavelength $\lambda$ of long-wave instability as given below:

$$
\lambda=\left[-\frac{8 \pi^{2} \gamma}{(\partial \phi / \partial h)}\right]
$$

The other limitation is on aspect ratio of dewetted structure due to very small contact angle. To overcome the limitations, polymer thin films were destabilized by immersing into mixture of organic solvent methylethylketone (MEK) and water at room temperature [18]. This type of immersing of polymer thin film into solvent leads to selective diffusion of solvent molecules into the polymer matrix, which causes the lowering of its glass transition temperature $\left(T_{\mathrm{g}}\right)$ below the room temperature and thus, leads the instability and dewetting of the film, which finally, results in the formation of microlenses.

The number density of holes $\left(N_{\mathrm{H}}\right)$ is governed by wavelength $(\lambda)$ of long-wave instability and is proportional to $\lambda^{-2}$ (or $h^{-4}$ ), which means the number density of holes or dewetting decreases with increase in the thickness of the film [18-21]. From the above discussion, we depict that the films with thickness below $100 \mathrm{~nm}$ become unstable and break up into droplets due to complex interactions between interfacial energy and van der Waals forces [21]. As the disjoining pressure increases, the interaction energy per unit area increases, wave length of instability decreases, i.e., there is increase in the number density of holes which increases dewetting and thus, enhances droplet formation.

\section{Experimental}

Dewetting of polymer thin films is an established and well known process as described by Verma et al [19]. For fabrication of microlenses of polystyrene (PS) or poly(methyl methacrylate) (PMMA), we followed the same procedure. PS and PMMA with molecular weights 280,000 and 120,000 $\mathrm{g} \mathrm{mol}^{-1}$, respectively, were purchased from Sigma Aldrich. The microlens fabrication process involves cleaning of glass substrate, preparation of transparent PS and PMMA solutions, spin coating of PS and PMMA solutions on glass substrate, preparation of dewetting solution, dewetting of PS and PMMA thin films and formation of PS and PMMA microlenses. The glass substrates were thoroughly cleaned in soap solution, followed by cleaning in organic solvents such as trichloroethylene (TCE), acetone and methanol for $10 \mathrm{~min}$ each. Then, the substrates were rinsed thoroughly with deionized (DI) water and dried.

To make microlenses from polymer thin films, PS and PMMA solutions were made with different concentrations (0.2, 0.3 and $0.4 \%$ in toluene). These solutions of PS and PMMA in toluene were ultrasonicated for $15 \mathrm{~min}$ and kept overnight, which were then again ultrasonicated for $15 \mathrm{~min}$ before use. Clear solutions of PS and PMMA were obtained. The PS and PMMA solutions ( $0.4 \%$ in toluene) were spincoated on glass substrates at $2000 \mathrm{rpm}$. Thickness of both PS and PMMA thin films were found to be $\sim 25 \mathrm{~nm}$. These polymer thin films were then kept for $2 \mathrm{~h}$ under a laminar clean air bench. With respect to annealing of films, we made a significant departure from the process reported by Verma et al [20]. Instead of annealing the films at $60^{\circ} \mathrm{C}$ in vacuum oven for $12 \mathrm{~h}$, we found that the process can be made much shorter. After optimizing the process with respect to evolution of the microlenses, we annealed the polymer thin film at $110^{\circ} \mathrm{C}$ for 30 min on a hot plate, which was found sufficient to drive off all the toluene from the film. After $30 \mathrm{~min}$, these substrates were removed from hot plate and allowed to cool at room temperature. The cooled down films were kept in dewetting solution for $10 \mathrm{~min}$. Dewetting solution is a homogeneous mixture of DI, MEK and acetone in the ratio of 15:7:3. When polymer thin film was kept into dewetting solution, film rupture took place due to instability, which resulted in the formation of microlenses of sub-micrometre dimensions.

Area fraction of coverage by microlenses with films of PS or PMMA is small, leaving large areas with no microlenses. 
So, in some cases, to enhance the area fraction of microlenses, we prepared multilayer of polymers to form microlenses. In this strategy, first PS and PMMA both the solutions were made in same solvent toluene. Then, PS was spin-coated on a glass slide at $3000 \mathrm{rpm}$ and microlenses were formed as mentioned earlier. Then, second layer of PS was again coated on top of the first layer of microlenses before carrying out dewetting of the second layer. Same process was followed for PMMA solution. But, using the same solvent for the second layer may dissolve the underlying microlenses.

Area coverage of the microlenses can be increased by multiple layer coatings. In this process, first PMMA is dewetted resulting in an array of isolated PMMA droplets. This sample with PMMA droplets is then surface-treated with octadecyltrichlorosilane (OTS), and subsequently, a PS solution is spin-coated on it, this also undergoes dewetting. This dewetted PS also forms the microlenses in between PMMA microlenses, and hence area fraction can be increased [22].

In the approach we followed, different solvents for both PS and PMMA were taken to prevent dissolution of PS and PMMA layers into each other and multilayer was formed by coating PMMA on PS or vice versa. To protect PS microlenses with toluene solvent, PMMA solution was made in acetic acid, while to prevent underlying, PMMA microlenses cast from toluene solvent, PS solution was made in cyclohexane. PS and PMMA were spin-coated alternatively.

We measured changes in luminance of a white LED with and without microlenses attached to it. The OLEDs made in laboratory are prone to luminance degradation, prohibiting use of same OLED in our study over a period of time. Hence, to maintain consistency of the light source over all the measurements, a stable source is needed, such as commercial LED. But, to emulate a diffused OLED light source, normally close to Lambertian, we render commercial LEDs into an equivalent diffused light source; a sand-blasted glass was placed on one side after the LED. The microlenses are made on another glass surface.

A robust setup for luminance measurement of bright white LED was made (figure 1). In this setup, a bright white LED was kept on a stand. Then, a plain glass with alignment mark was placed at a distance of $36 \mathrm{~cm}$ from LED. A sand-blasted glass was used as a diffuser and kept in front of glass with alignment mark at a distance of $0.9 \mathrm{~cm}$ to make a diffused light source. This would mimic light emanating from an OLED. Then, a glass substrate, on which PS microlenses were fabricated, was pasted on top of the sand-blasted glass slide with the help of an index matching fluid. The final glass surface is flat and the light escaping from it is a diffused light source similar to that from an OLED.

To detect small changes, first, the source LED was stabilized by keeping it in on-state for $1 \mathrm{~h}$. First, luminance at the plain glass surface was measured using a Minolta spectroradiometer, followed by a similar measurement after attaching the glass with microlenses and maintaining the place of measurement with the help of alignment marks. The difference of luminance between the two measurements provides luminance enhancement. However, at the end of experiment, the luminance at plain glass was again measured to ensure that LED was stable.

\section{Results and discussion}

This section is divided into two parts. In first part, we examine the time evolution of microlenses fabricated by the dewetting process, followed by the second part, which involves luminance measurement of white LED without and with microlenses. Time evolution study of microlenses was studied with PS thin film ( $0.4 \%$ in toluene) at different time intervals which shows the evolution of droplets in time, resulting in microlenses. Figure 2 shows the optical micrograph of the microlenses, which were fabricated by dewetting of $25 \mathrm{~nm}$ PS thin film (from $0.4 \mathrm{wt} \%$ in toluene). Measured average diameter and area fraction of the microlenses are $0.68 \mu \mathrm{m}$ and $20 \%$, respectively.

The sequential optical images of another sample of microlenses at different time intervals would demonstrate the time evolution of microlenses, specifically formation of holes in the films, limiting the polymer to the dark lines. These holes grow with time and then start coalescing to form a network. This network then breaks into droplets, which are the microlenses.

From these images, it can be inferred that once dewetting of polymer thin film starts, formation of holes on thin film takes place and these holes start coalescing after 4 min (figure 3a); the lighter-shaded regions are the holes between PS networks. Along with time, these holes grow in size and start coalescing, but a network of polymers still visible (figure $3 \mathrm{~b}$ ) in $7 \mathrm{~min}$. After a sufficient time interval of $10 \mathrm{~min}$, this network (darker lines of PS) breaks into isolated droplets, which are called microlenses (figure 3c). SEM images of time evolution of microlenses is shown in figure $4 a-c$.

With incorporation of these lenses, the light extraction is measured through luminance. Total internal reflection (TIR) takes place at substrate-air interface of an OLED, which is one of the limiting factors for improvement in light-extraction efficiency. This effect results in $\sim 20 \%$ loss in extraction of light. To reduce TIR at the substrate-air interface from where the light leaves the device, microlenses are applied on the front side of the device. These microlenses increase the cone of escape of light, thereby improving light extraction.

To evaluate the light extraction capability of these microlenses, they were attached to the flat side of the glass. In practice, these microlenses would be fabricated on the OLED glass substrate itself, but in this case, where microlenses are prepared on a separate glass, these are attached with an index matching fluid (as shown in figure 2) to minimize the loss due to refractive index mismatch.

Three such fluids, namely the common glue (fevikwik), xylene and glycerine, were examined in this work. To select one, transmittance was measured after joining two glass slides with one of these three liquids in between them. 

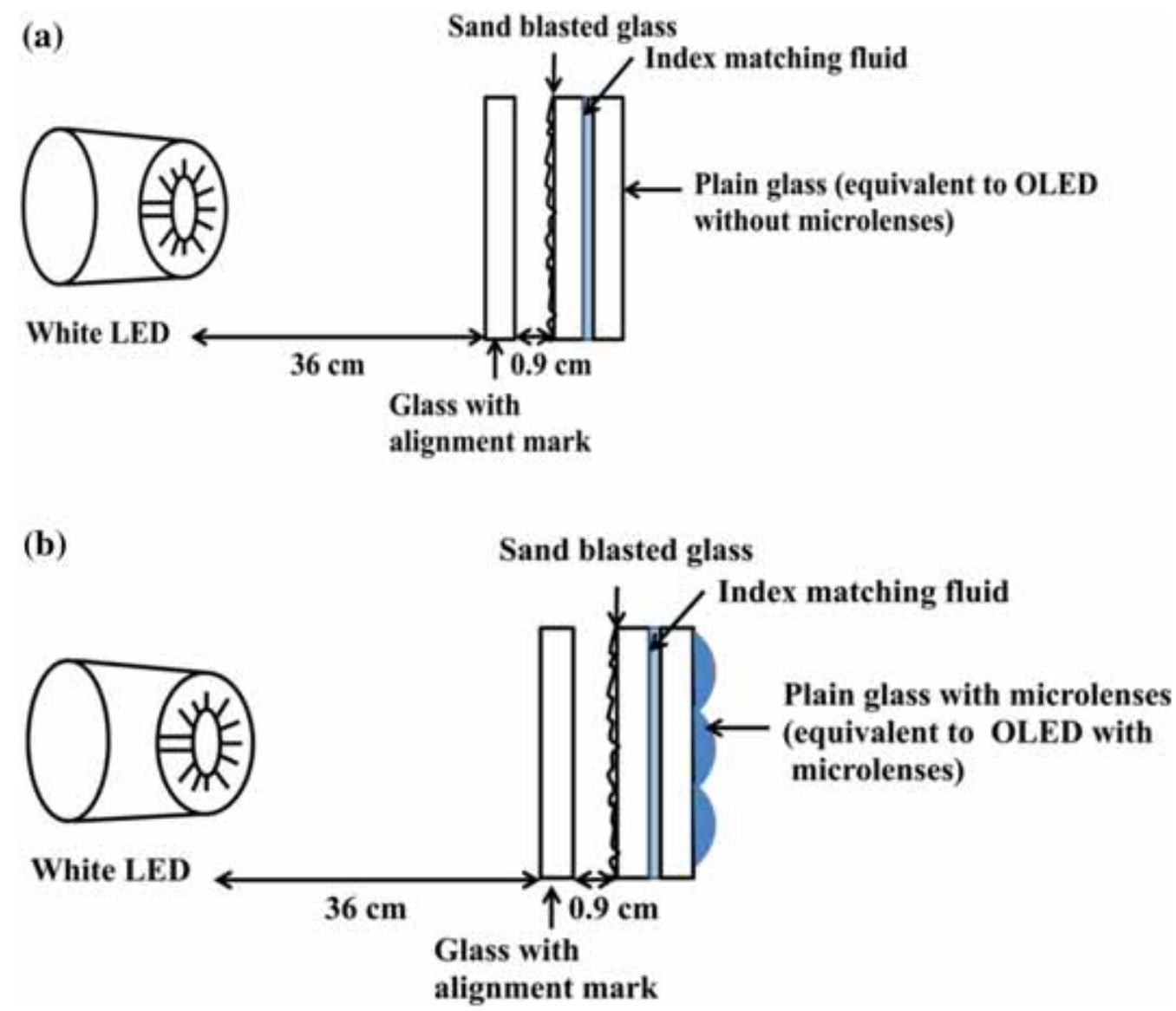

Figure 1. Schematic diagram of luminance measurement setup (a) with microlenses and (b) without microlenses.

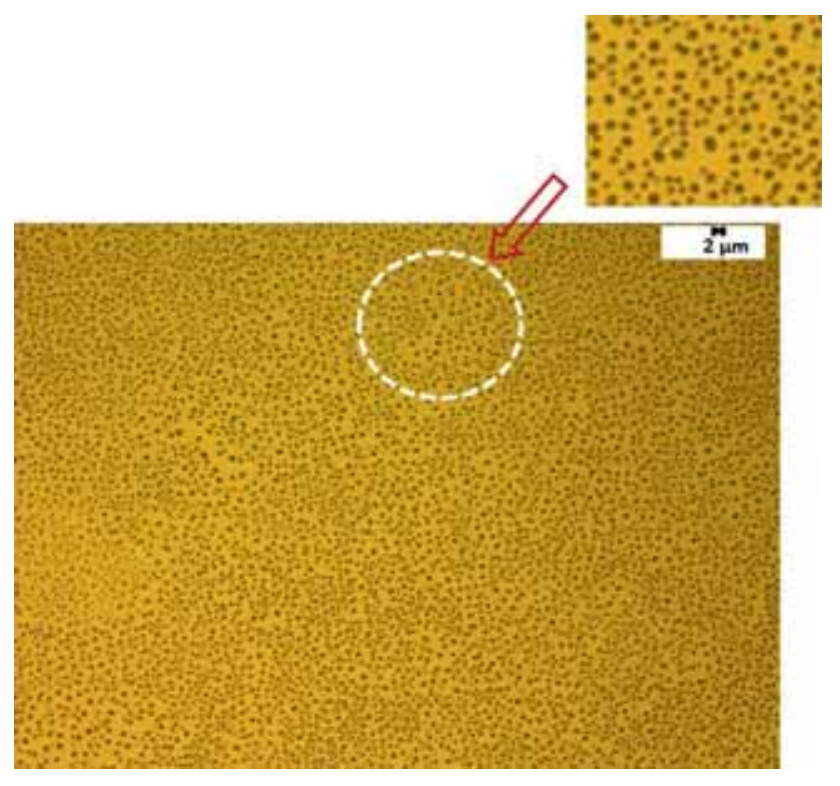

Figure 2. Optical micrograph of the polystyrene (PS) microlens droplets fabricated by dewetting. Inset shows magnified image of the fabricated microlenses.
All the liquids work very well as index matching fluid and transmittance is nearly $90 \%$ in all the cases (figure 5). Thus, glycerine which is the least toxic among the mentioned fluids was selected as index matching fluid between two glass slides; fevikwik binds the slides so tightly that these slides cannot be separated again, while xylene is inflammable and has acute toxicity, so it may cause health hazards.

\subsection{Luminance measurements}

To study the effect of microlenses on luminance enhancement of white LED, we measure luminance of LED after applying microlenses. The luminance improvement thus calculated is enumerated in table 1 . The PS microlenses that were made with $0.4 \%$ PS in toluene, in fact, reduced luminance. The decrease in the luminance at $0.4 \%$ concentration was caused due to increased haziness in the film at this concentration. Hence, we first examine the effect of change in the PS concentration in the solution.

From table 1, it can be inferred that out of all the different concentrations of PS in toluene solution, luminance enhancement was found only for $0.3 \%$ PS solution in toluene. 

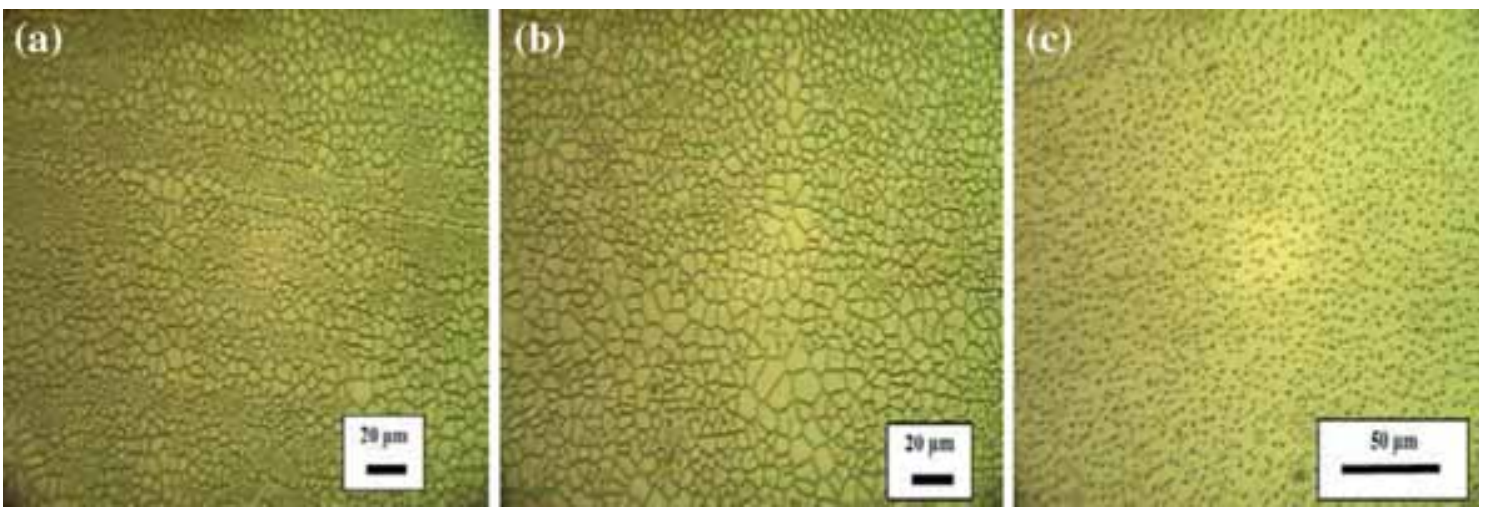

Figure 3. Time evolution of $25 \mathrm{~nm}$ polystyrene (PS) thin film on glass substrate under MEK solution. Morphology of PS thin film is taken after (a) 4 min as partially coalesced holes, (b) 7 min as completely coalesced holes forming a network and (c) $10 \mathrm{~min}$ as isolated polymer droplets.
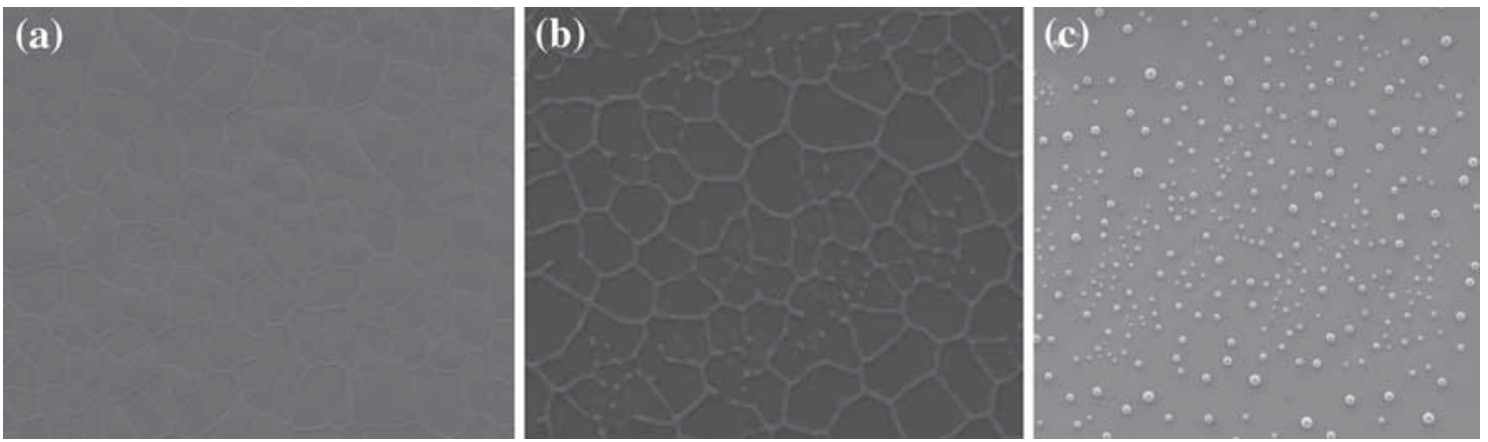

Figure 4. SEM images of time evolution of polystyrene (PS) thin film on glass substrate under MEK solution after (a) $4 \mathrm{~min}$ as partially coalesced holes, (b) $7 \mathrm{~min}$ as completely coalesced holes forming a network and (c) $10 \mathrm{~min}$ as isolated polymer droplets (images are taken at a magnification of 20).

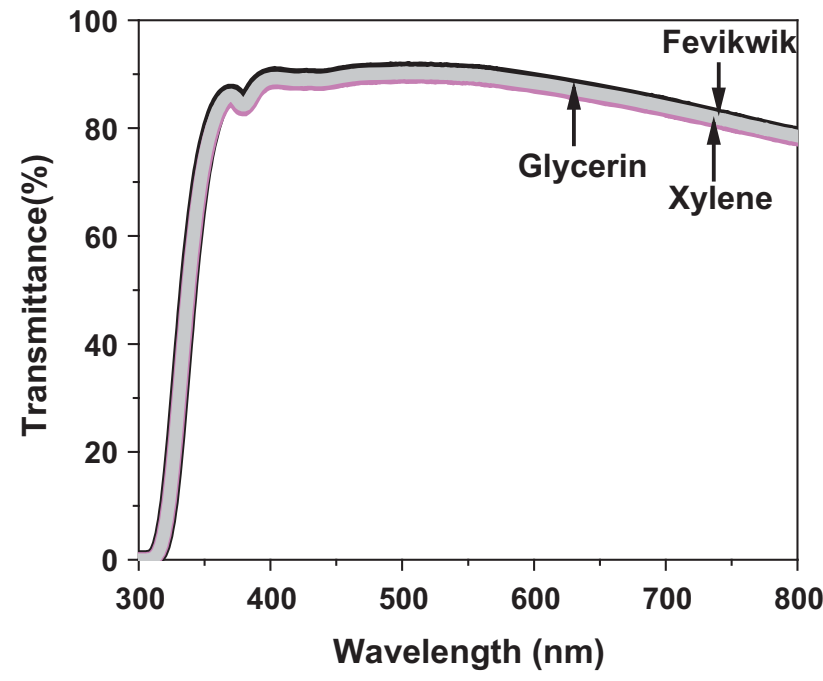

Figure 5. Transmittance measurements of two glass slides joined by fevikwik, xylene and glycerine as index matching fluids.
In this case, the film thickness was $20 \mathrm{~nm}$, lenses were of diameter $1.1 \mu \mathrm{m}$ and are fraction covered by microlenses, which was $25 \%$. For $0.2 \%$ concentration of PS in toluene, dewetting was not proper.

Since the study of the effect of various microlens parameters such as diameter and area fraction on light extraction from LED was not done systematically, an attempt to systematically study these parameters is made by varying the diameter and area fraction of microlenses. In doing so, concentration of PS in toluene maintained at $0.3 \%$. Average diameter of microlenses increases with film thickness, while number density of droplets decreases with film thickness. To increase area fraction or to increase number density of microlenses two strategies were evaluated as below:

(i) Multiple-coating and dewetting with different combinations of polymers and solvents.

(ii) Film thickness is modified by controlling spin-coating speed. 
Table 1. Luminance enhancement with microlenses made by different concentrations of polystyrene (PS) in toluene.

\begin{tabular}{lcc}
\hline$\%$ PS solution in toluene & Thickness of the film $(\mathrm{nm})$ & $\%$ Improvement in luminance \\
\hline 0.2 & 18.2 & Improper dewetting \\
0.3 & 19.9 & 3.83 \\
0.4 & 25.7 & -7.93 \\
\hline
\end{tabular}

Table 2. Measurement of diameter and area fraction of microlenses with spin speed and thin film thickness.

\begin{tabular}{lccc}
\hline Sample & Average diameter $(\mu \mathrm{m})$ & Lens coverage area fraction $(\%)$ & Thickness of the film $(\mathrm{nm})$ \\
\hline PS/PS (3000 rpm) & 0.32 & 20.8 & 39.8 \\
PMMA/PMMA (3000 rpm) & 0.35 & 18.5 & 40.5 \\
PS/PMMA (3000 rpm) & 0.98 & 28.5 & 25.3 \\
PMMA/PS (3000 rpm) & 1.55 & 25.4 & 29.5 \\
PS (500 rpm) & 2.36 & 19.4 & 40.2 \\
PS (1000 rpm) & 1.29 & 24.8 & 30.1 \\
PS (3000 rpm) & 1.06 & 34.6 & 19.8 \\
PMMA (500 rpm) & 0.91 & 21.5 & 32.5 \\
PMMA (1000 rpm) & 0.49 & Improper dewetting & 24.3 \\
PMMA (3000 rpm) & & 26.1 & \\
\hline
\end{tabular}
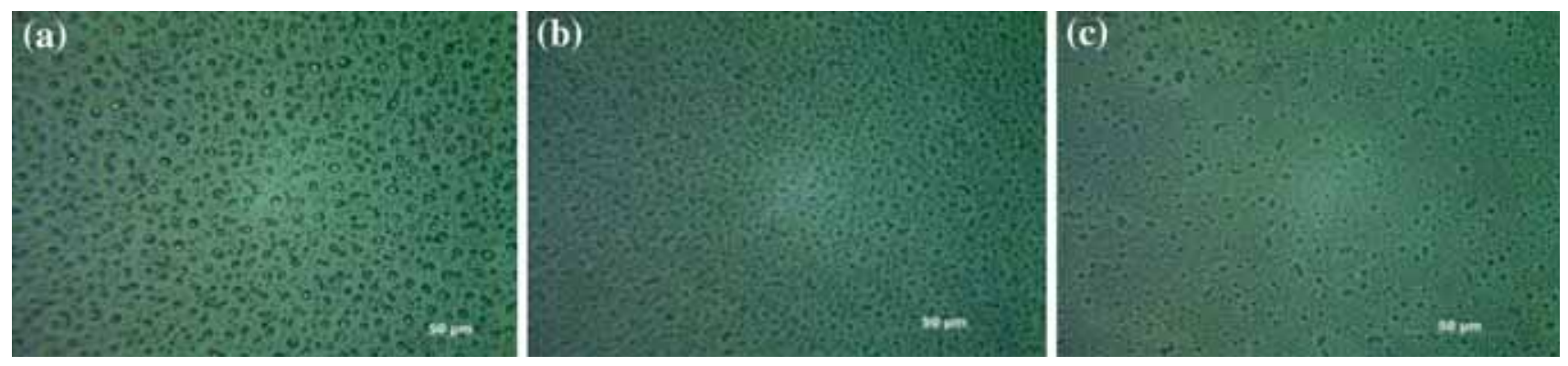

Figure 6. Optical images of microlenses fabricated using PS film with different thicknesses: (a) 40, (b) 30 and (c) $20 \mathrm{~nm}$.

After the formation of lenses with $0.3 \%$ PS in toluene, spin-coated at $3000 \mathrm{rpm}$, another layer of the same solution was coated, the area coverage was $20.8 \%$, which is even slightly lower than that for single layer. Similar observation applied to dual layer PMMA microlenses. The reason was dissolution of PS and PMMA layer during second layer coating under same solvent (first two rows in table 2).

Hence, to enhance area fraction of microlenses, another layer of PMMA was coated above PS microlenses cast from toluene solvent and that layer was also dewet to make microlenses. In this case, to protect the PS microlenses, the PMMA solution was made in acetic acid. Conversely, when PMMA microlenses were made first, the solvent used was toluene, but now PS solution was made in cyclohexane. In the case of PMMA on top of PS, the area fraction of microlenses is $28 \%$. Similarly, for PS on top of PMMA, the area coverage is $25 \%$, not significantly different from single layer microlenses. In other words, the attempt to increase area density of microlenses, the multilayer strategy was not found suitable.

Alternatively, to modify the diameter of the microlenses, in single layer of either PS or PMMA, the thickness was varied through manipulating the spin-coating speeds, specifically, 500, 1000 and $3000 \mathrm{rpm}$. Thicknesses of these films for PS were found to be 40,30 and $20 \mathrm{~nm}$, respectively. The results of all the strategies followed for the improvement of area fraction and diameter of microlenses are summarized in table 2 . The area fraction of microlenses increased maximum up to $34 \%$ with polymer thin films with different thicknesses.

From table 2, it is concluded that the maximum improvement in area fraction can be achieved by varying the spin speed of PS thin film, i.e., by making the thin film of different 
Table 3. Measurement of diameter and area fraction of microlenses with spin speed and thin film thickness.

\begin{tabular}{lccc}
\hline Thickness of PS thin film $(\mathrm{nm})$ & Avg. diameter $(\mu \mathrm{m})$ & Area fraction $(\%)$ & $\%$ Improvement \\
\hline 40 & 2.36 & 19.4 & $3.89 \pm 0.17$ \\
30 & 1.29 & 24.8 & $5.72 \pm 0.05$ \\
20 & 1.06 & 34.6 & $6.76 \pm 0.07$ \\
\hline
\end{tabular}

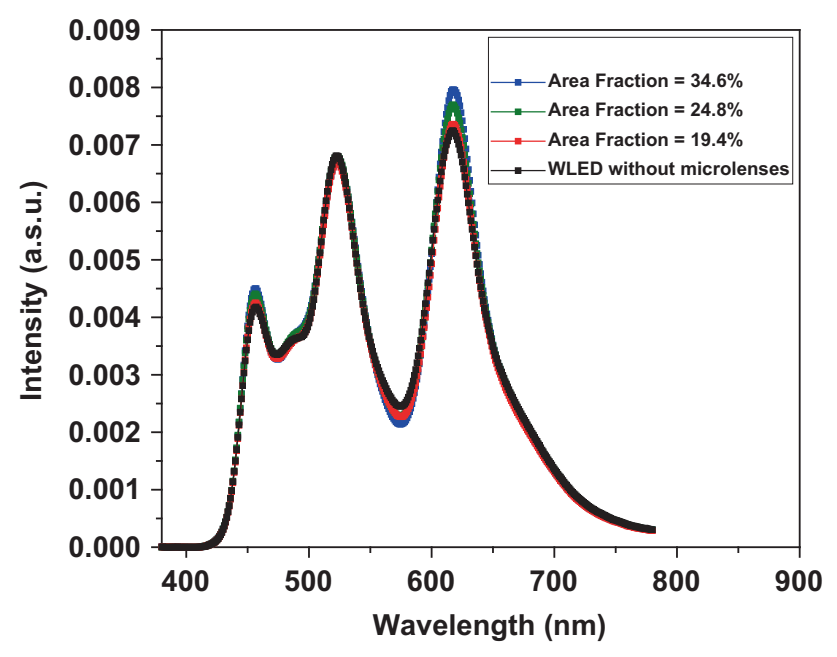

Figure 7. Electroluminescent (EL) spectrum of white LED without and with microlenses. Plot depicts the increment in EL spectrum upon increase in area fraction of the microlenses.

thicknesses. After these experiments, we have chosen the PS films of different thicknesses to study the extraction efficiency of LED.

Figure 6 shows the optical images of PS microlenses fabricated with films of different thicknesses varying from 20 to 40 $\mathrm{nm}$. From this figure, it is depicted that if the spin speed of film is increased, the film thickness decreases, and so the average diameter, but the area fraction increases. Here, thicknesses of the films were measured using thickness profilometer. Effect of different microlenses parameters on luminance was measured using Minolta spectroradiometer. Measured values of film thickness, microlens diameter, area fraction and luminance are shown in table 3 .

From table 3, it can be depicted that as the thickness of the PS film decreases, average diameter decreases and the area function increases, which is in agreement with the proposed theories cited in literature. The improvement in the luminance can be attributed to the increase in the area fraction of the microlenses, which improves the light extraction.

Figure 7 shows that the electroluminescent (EL) spectrum of white LED is a very strong function of area fraction of microlenses. It can be inferred from this plot that the EL of white LED increases as we increase the area fraction of microlenses.

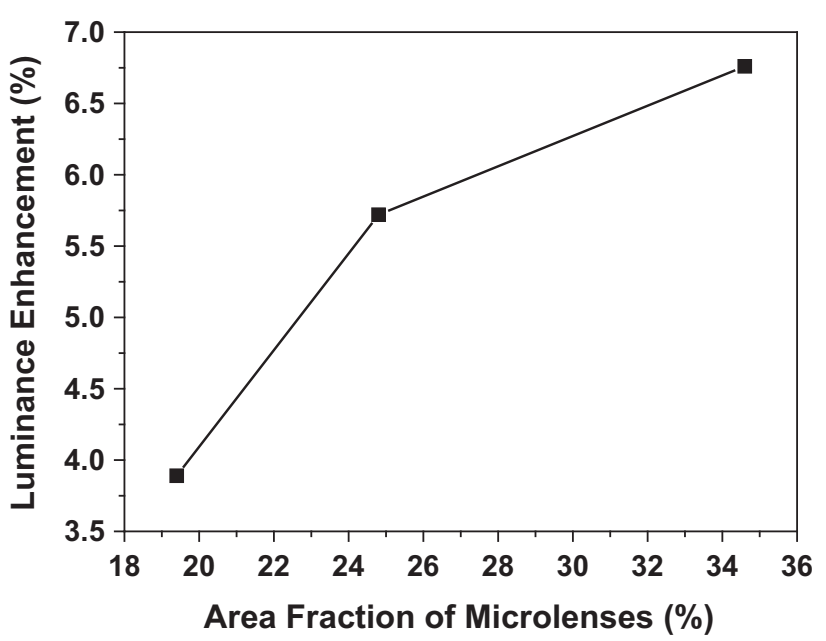

Figure 8. Area fraction of microlenses vs. luminance enhancement.

From figure 8 , it can be depicted that as the area fraction of microlenses increases, the light outcoupling from LED increases, which is an evidence for EL increment of white LED with the application of microlenses. We have also observed that the enhancement is prominent for microlenses with smaller dimensions as it is easier to make hemispherical as compared to large size microlenses.

\section{Conclusions}

In this paper, we have successfully demonstrated an alternative way to fabricate microlenses, which is cost effective and not cumbersome. Several conclusions can be made based on the experimental results and discussions in the paper: (i) an inexpensive and effortless methodology for fabrication of microlenses, i.e., dewetting technique is described, (ii) we can get rid of conventional photolithography by using dewetting of polymers, (iii) dimensions of the microlenses fabricated by dewetting is very small as compared to pixel size of the display, hence, by using these sub-micrometre lenses, we can also get rid of image blurring caused by larger microlenses in an OLED display, (iv) dewetting can be used to fabricate microlens of the order of sub-micrometre (1-2 $\mu \mathrm{m})$, and (v) from the above results, we can conclude that outcoupling 
efficiency enhances as area fraction of microlens increases. However, area coverage still needs to be improved.

\section{Acknowledgements}

We would like to thank Prof Ashutosh Sharma and Ms Priyanka Sachan, both at IIT Kanpur, for initiating us in the process that they earlier developed to create instability in thin films.

\section{References}

[1] Saxena K, Jain V K and Mehta D S 2009 Opt. Mater. 32 221

[2] Möller S and Forrest S R 2002 J. Appl. Phys. 913324

[3] Lin H Y, Ho Y H, Lee J H, Chen K Y, Fang J H, Hsu S C et al 2008 Opt. Express 1611044

[4] Melpignano P, Biondo V, Sinesi S, Gale M T, Westenhöfer S, Murgia M et al 2006 Appl. Phys. Lett. 88153514

[5] Peng H J, Ho Y L, Qiu C F, Wong M and Kwok H S 2004 SID 04 DIGEST 158

[6] Peng H, Ho Y L, Yu X J, Wong M and Kwok H S 2005 J. Display Technol. 1278
[7] Eom S H, Wrzesniewski E and Xue J 2011 Org. Electron. 12 472

[8] Wrzesniewski E, Eom S H, Cao W, Hammond W T, Lee S, Douglas E P et al 2012 Small 82647

[9] Li X H, Song R, Ee Y K, Kumnorkaew P, Gilchrist J F and Tansu N 2011 IEEE Photon. J. 3489

[10] Li X H, Ee Y K, Song R and Tansu N 2011 CLEO: 2011, Optical Society of America, Baltimore, Maryland, p CML4

[11] Wei M K and Su I L 2004 Opt. Express 125777

[12] Wei M K, Su I L, Chen Y J, Chang M, Lin H Y and Wu T C 2006 J. Micromech. Microeng. 16368

[13] Wei M K, Lin H Y, Lee J H, Chen K Y, Ho Y H, Lin C C et al 2008 Opt. Commun. 2815625

[14] Lee J H, Ho Y H, Chen K Y, Lin H Y, Fang J H, Hsu S C et al 2008 Opt. Express 1621184

[15] Yang J P, Bao Q Y, Xu Z Q, Li Y Q, Tang J X and Shen S 2010 Appl. Phys. Lett. 97223303

[16] Oslanec R, Costa A C and Composto R J 2000 Macromolecules 335505

[17] Mukherjee R and Sharma A 2015 Soft Matter 118717

[18] Verma A and Sharma A 2011 Macromolecules 444928

[19] Verma A and Sharma A 2010 Adv. Mater. 225306

[20] Choi S H 2006 PhD Thesis, University of Akron

[21] Mukherjee R, Das S, Das A, Sharma S K, Raychaudhuri A K and Sharma A 2010 ACS Nano 43709

[22] Bhandaru N, Das A, Salunke N and Mukherjee R 2014 Nano Lett. 147009 